\title{
Low-income women in California may be at risk of inadequate folate intake
}

by Emily R. Cena, Amy Block Joy,

Karrie Heneman and Sheri Zidenberg-Cherr

Folate plays a major role in preventing neural tube defects in the developing fetus, as well as in reducing the risks of cardiovascular disease, certain types of cancer and some mental health problems. We assessed the folate intakes of socioeconomically disadvantaged women of childbearing age participating in California's Food Stamp Nutrition Education program. Of 195 women studied, $59 \%$ failed to meet the Institute of Medicine's folate intake recommendations for women capable of becoming pregnant. We found significant differences among the ethnic groups studied: $45 \%$ of Hispanic, $65 \%$ of white and $77 \%$ of black women did not meet the recommendation for synthetic folic acid intake. This study supports the need for developing targeted nutrition-education lessons focusing on the importance of adequate folate consumption.

Colate is a B vitamin required for - critical bodily functions such as DNA synthesis and repair, and amino acid metabolism. This vitamin is found in foods such as liver, lentils and other legumes, oranges and orange juice, and dark-green leafy vegetables. It is well established that folate deficiency causes macrocytic, hyperchromic anemia, a red-blood-cell condition that causes weakness, fatigue, loss of appetite and confusion. More recently, suboptimal folate levels have been associated with cardiovascular disease (Boushey et al. 1995), certain types of cancer (such as colon and pancreatic) (Jennings 1995) and some mental health disorders, including depression and dementia (Bodnar and Wisner 2005; Campbell et al. 2005).

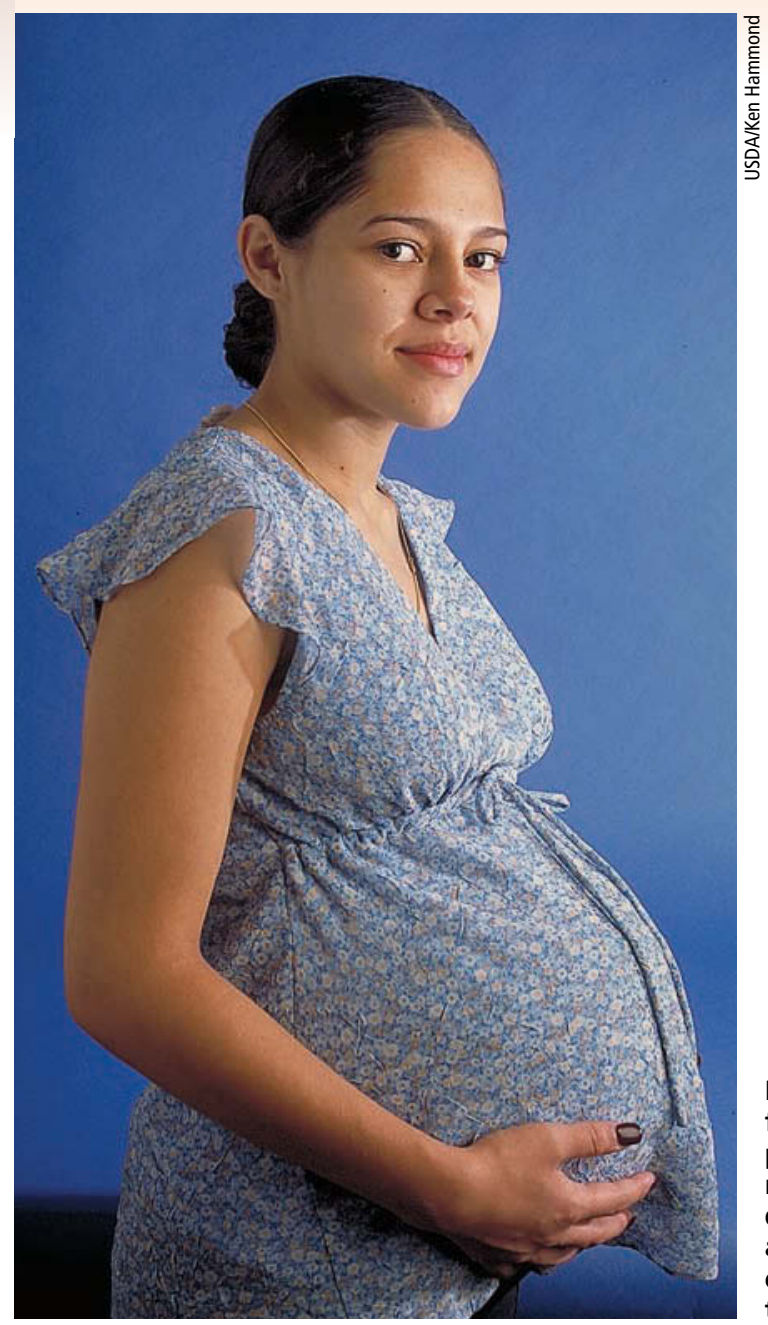

Daily folic acid intake is important for all women who could become pregnant, because it reduces the risk of neural tube defects in the developing fetus. A daily folic acid-containing supplement is an effective way for women to meet the folic acid recommendation.

Low folate levels are also associated with birth defects. Women deficient in this vitamin are more likely to have babies with neural tube defects such as spina bifida or anencephaly (MRC 1991). These defects occur during the first 4 weeks of embryonic development due to incomplete closure of the neural tube, which ultimately becomes the brain and spinal cord. Neural tube defects can cause physical abnormalities, developmental problems, partial or complete paralysis, and may even cause death (before or after birth).

Adequate folate intake can reduce the risk of neural tube defects by as much as $72 \%$, according to a randomized, doubleblind vitamin supplementation trial involving 1,817 women of childbearing age from seven countries (MRC 1991). In an effort to reduce the incidence of neural tube defects, the United States has required the mandatory fortification of enriched grains with synthetic folic acid (SFA) since 1998; some other countries also mandate folate fortification, including Canada and Chile.

\section{Folate recommendations}

There are currently two types of folate in the U.S. food supply: (1) SFA and (2) folate that occurs naturally in a limited number of foods, such as spinach and beans. SFA is added to enriched grain products, including ready-to-eat breads and breakfast cereals, and is also found in folic acid-containing vitamin supplements. The primary difference between these two types of folate is that SFA is more easily digested and 


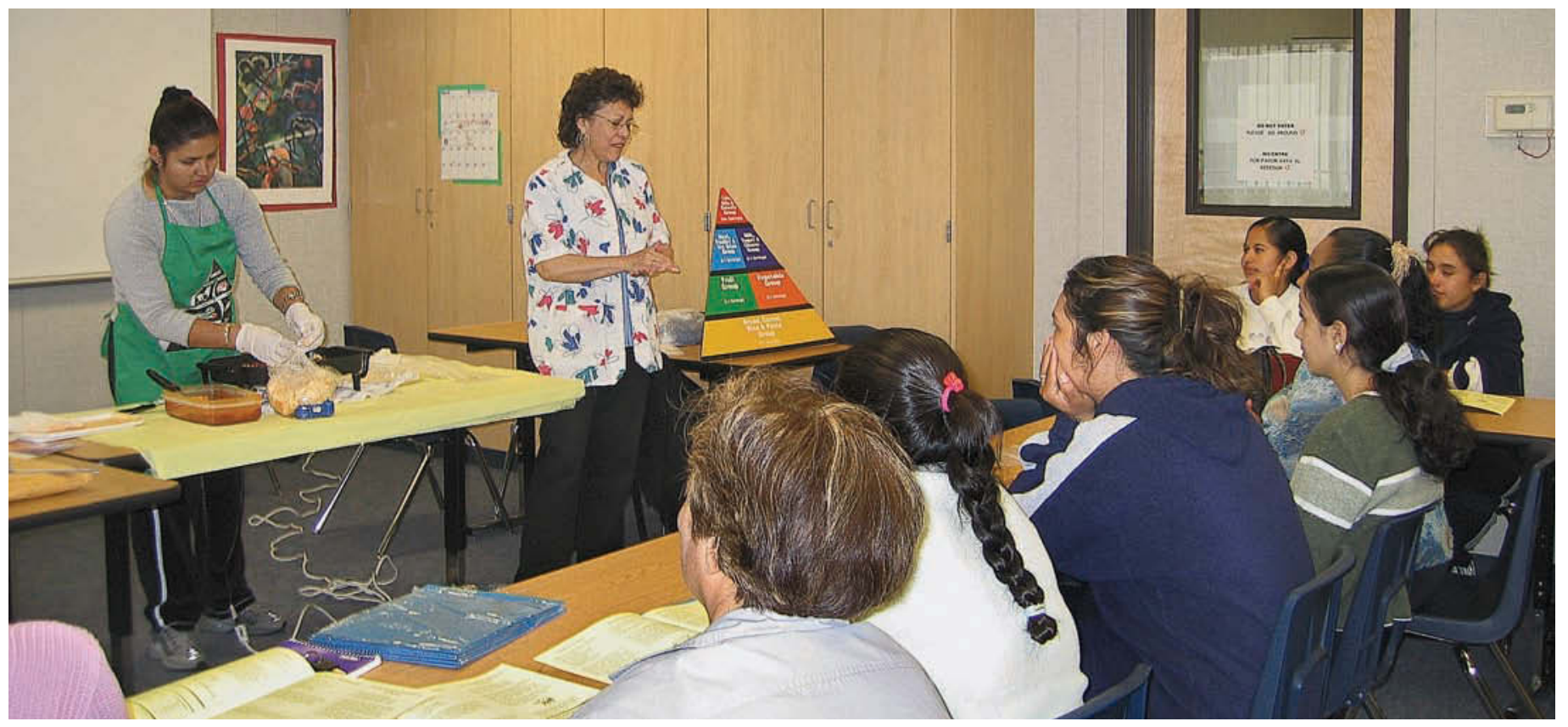

The Food Stamp Nutrition Education program teaches low-income people how to purchase and prepare healthy foods. In this study, researchers evaluated the folate intakes of female program participants. Yolanda Lopez, FSNE program nutrition educator in Fresno County (right), and a volunteer conduct a cooking demonstration.

absorbed, making it more readily available to the body's tissues (bioavailable) (IOM 1998).

To account for the difference in bioavailability between types of folate, the intake of this vitamin is typically quantified with a standard unit called the dietary folate equivalent (DFE). In 1998, the Food and Nutrition Board of the Institute of Medicine (an independent arm of the National Academy of Sciences) published the Dietary Reference Intakes for folate (IOM 1998). They include a Recommended Dietary Allowance (RDA), a Tolerable Upper Intake Level (UL), and a special recommendation for women capable of becoming pregnant to consume at least $400 \mu \mathrm{g}$ SFA/day, in addition to the natural food folate found in a varied diet (see glossary).

\section{Impact of fortification uneven}

Research suggests that the U.S. folate fortification program has been only partially successful. On the positive side, the general population has significantly higher mean folate levels in red blood cells and serum, according to a comparison of National Health

\section{Glossary}

Block DFE screener: An instrument that quickly estimates the usual folate intake from 21 food and supplement sources. The screener identifies individuals who may be at risk of low folate status, especially in low-income populations.

Dietary folate equivalent (DFE):

The standardized unit for measuring folate intake: 1 microgram $(\mu \mathrm{g}) \mathrm{DFE}=$ $1 \mu \mathrm{g}$ natural food folate $=$

$0.5 \mu \mathrm{g}$ SFA from a supplement that is taken on an empty stomach $=0.6 \mu \mathrm{g}$ SFA taken with food or from a fortified food source.

Dietary Reference Intakes (DRIs): Recommendations by the Institute of Medicine for nutrient intakes, which can be used for planning and assessing diets. The DRIs for folate include a Recommended Dietary Allowance, Tolerable Upper Intake Level, and a special recommendation for women capable of becoming pregnant.

Folate: $B$ vitamin required by the body for a variety of functions, including DNA synthesis and repair; includes naturally occurring food folate and SFA.

Recommended Dietary Allowance (RDA): The intake level for a nutrient that the Institute of Medicine considers sufficient to meet the needs of almost all healthy people of a given age and gender. For folate, the RDA for adults is $400 \mu \mathrm{g}$ DFE per day, based on the amount of dietary folate required to maintain normal blood concentrations of certain folate status indicators.

Special recommendation for women of childbearing age: A recommendation for women capable of becoming pregnant set forth by the Institute of Medicine, in addition to the RDA. In order to reduce the risk of giving birth to a child with a neural tube defect, women of childbearing age are recommended to consume $400 \mu \mathrm{g}$ SFA per day, in addition to the natural food folate supplied by a varied diet.

Synthetic folic acid (SFA): A human-made form of folate found in fortified grain products and vitamin supplements. The bioavailability of SFA is greater than that of natural food folate.

Tolerable Upper Intake Level (UL): The highest daily intake level for a nutrient that is likely to be safe for almost all healthy people of a particular age and gender. For folate, the UL for adults is $1,000 \mu \mathrm{g}$ SFA per day, regardless of the natural food folate consumed. This value is based on the possibility that very high intakes of SFA from supplements and fortified foods might mask a vitamin B-12 deficiency. 


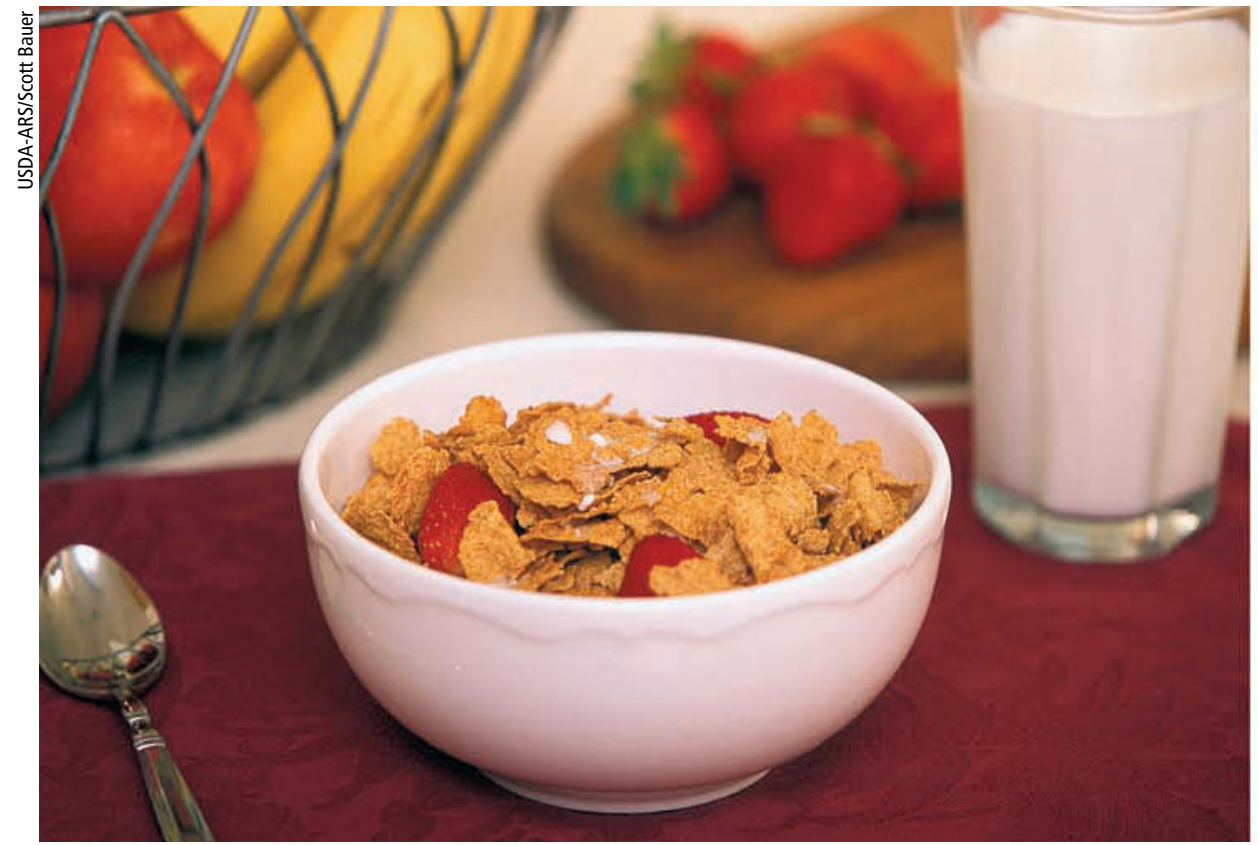

and Nutrition Examination Surveys (NHANES) before and after the fortification program began (1988-1994 and 1999-2000, respectively) (CDC 2002). In addition, there were $26 \%$ fewer neural tube defects 2 years after the program began than 3 years before (2000 and 1995, respectively) (CDC 2004). While this is a noteworthy improvement, it is still far less than the $72 \%$ reduction in neural tube defects expected if all women of childbearing age consumed adequate amounts of folate.

Moreover, other studies suggest that certain population subgroups may still be at risk. For example, the NHANES data indicates that non-Hispanic black women have $23 \%$ lower red-blood-cell folate levels and $26 \%$ lower serum folate levels than non-Hispanic white women (CDC 2002). In addition, socioeconomically disadvantaged women had $16 \%$ lower red-blood-cell folate levels and $24 \%$ lower serum folate levels than their socioeconomically advantaged counterparts, according to a study in Southern California (Caudill et al. 2001).

\section{Assessing folate intake}

The purpose of our study was to assess folate intake among low-income, food stamp-eligible $(\leq 130 \%$ of federal poverty level) women of childbearing age in California. In California, the Food Stamp Nutrition Education (FSNE) program serves approximately 138,000 food stamp-eligible families each year by providing nutrition education and skills training about selecting, purchasing and preparing healthy foods. Funding is provided via an interagency agreement among the U.S. Department of of Social Services and UC Davis.

\section{Participants and instruments.}

During spring 2005, 211 women participating in California's FSNE program were recruited for a cross-sectional survey of folate intake, from 12 counties: Alameda $(n=59)$, Amador $(\mathrm{n}=3)$, Calaveras $(\mathrm{n}=14)$, Fresno $(\mathrm{n}=$ 10), Los Angeles $(\mathrm{n}=11)$, Nevada $(\mathrm{n}=$ $12)$, Placer $(n=8)$, Riverside $(n=7)$, San Diego $(n=33)$, Shasta $(n=29)$, Trinity $(n=20)$ and Tuolumne $(n=5)$. Study participants were between 18 and 45 years old, nonpregnant and able to read and understand English or Spanish. The Institutional Review Board at UC Davis approved the study.

Study participants were asked to complete two forms: (1) a demographic survey, which was adapted from the FSNE program's Adult Family Record, and (2) the Block Dietary Folate Equivalents (DFE) Screener, a one-page, rapid screener developed to measure the usual intake of dietary folate in lowincome populations. The DFE Screener has previously been demonstrated to reflect red-blood-cell folate status in this population (Clifford et al. 2005).

The Block DFE screener is used to estimate folate intake in order to quickly and easily determine the risk of low folate status. However, it does not account for all sources of dietary folate. Rather, it measures the frequency of intake for the Agriculture, the California Department
Fortified breakfast cereals, broccoli and beans are good dietary sources of folate. More than half of the women studied were not getting enough folate from their diets or vitamin supplements.

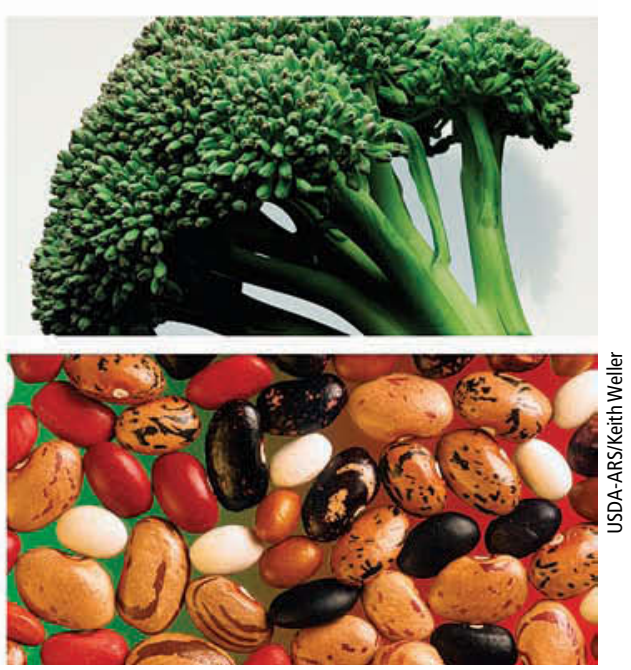

most common food sources of folate in the United States. A comprehensive, traditional, food-frequency questionnaire could be used for more quantitative folate-intake assessment, but we did not choose this option because of the substantial participant burden it entails, particularly in a low-income population. In addition, as with all studies of self-reported food intake, participants in our study may have under- or overreported their consumption of certain food groups. Finally, the food items included in the screener may not fully represent the dietary choices of different ethnic groups or individual variations.

The completed folate screeners were scanned and scored by Block Dietary Data Systems in Berkeley, California. Data from the demographic survey and folate screener were analyzed by independent $t$-tests, one-way analysis of variance and Tukey post-hoc multiple comparisons, using SPSS version 13.0.

Demographics. Of the 211 study participants, six were excluded due to incomplete surveys and 10 were excluded as outliers. As a result, 195 women were included in the final analysis. The average age $( \pm S E)$ of study participants was $33.5 \pm 0.5$ years old, with $45.1 \%(n=88)$ being white, $30.8 \%(\mathrm{n}=60)$ Hispanic and $13.3 \%$ $(\mathrm{n}=26)$ black. Due to small sample sizes of Asians, Pacific Islanders and Native Americans, these women as well as those who wrote in a different response were combined into an "other" category, which comprised 
TABLE 1. Estimated folate intake from food and supplement sources $(n=195)$ *

\begin{tabular}{|c|c|c|c|}
\hline Type of folate & $\begin{array}{c}\text { Mean intake } \pm \text { SE for } \\
\text { each folate typet }\end{array}$ & Bioavailability factor & $\begin{array}{l}\text { Mean DFE intake } \pm \text { SE } \\
\text { for each folate type }\end{array}$ \\
\hline & $\mu g$ & & $\mu g D F E$ \\
\hline $\begin{array}{l}\text { Naturally occurring } \\
\text { folate in foods }\end{array}$ & $198.0 \pm 14.6$ & 1.0 & $198.0 \pm 14.6$ \\
\hline SFA from fortified foods & $270.5 \pm 10.0$ & 1.7 & $459.9 \pm 17.1$ \\
\hline SFA from supplements & $148.8 \pm 16.6$ & 1.7 & $253.0 \pm 28.2$ \\
\hline
\end{tabular}

$6.7 \%(\mathrm{n}=13)$ of respondents. The remaining $4.1 \%(\mathrm{n}=8)$ did not report their ethnicity. Approximately twothirds $(71.3 \%)$ of the respondents completed the surveys in English.

\section{Folate consumption evaluated}

The Block DFE screener provided information about participants' intake levels of naturally occurring food folate, SFA from fortified foods and SFA from folic acid-containing vitamin supplements. Of the 195 participants, 41\% $(\mathrm{n}=80)$ reported taking a supplement containing folic acid at least twice per month. The mean estimated total intake of folate from all sources was 911 \pm 33 (mean \pm SE) micrograms $(\mu \mathrm{g}) \mathrm{DFE}$ per day (table 1). Of this, $78 \%$ (713 $\mu \mathrm{g}$ DFE) was in the form of SFA from fortified foods and supplements (table 1). Although the mean intake for total DFE exceeded the RDA, and mean total SFA intake exceeded the special recommendation for women of childbearing age to consume at least $400 \mu \mathrm{g}$ SFA per day, nearly $59 \%$ of the participants did not meet the latter recommendation (table 2).

After comparing the mean folate intakes to dietary recommendations, we tested for differences in intake according to demographic characteristics. One-way analysis of variance revealed significant differences in folate intake between ethnic groups (fig. 1). On average, Hispanic women consumed $48 \%$ more SFA than black women $(P<0.05)$. Additionally, Hispanic women consumed, on average, $28 \%$ more total DFE than white women, and $46 \%$ more total DFE than black women $(P<0.01)$. Even so, $45 \%$ of the Hispanic women in this study had usual SFA intakes below the recommendation. Suboptimal SFA intakes were more prevalent in the other two largest ethnic cat- egories, with $65 \%$ of white women and $77 \%$ of black women in this study failing to meet the SFA recommendation.

The mean intakes for women classified as "other" were not significantly different from those of the other three groups for either total DFE or SFA, possibly due to the small sample size. In addition, there were no significant differences in total dietary folate intake or SFA intake according to county of residence, language or age.

\section{Role of vitamin supplements}

More than half of the women participating in our study had suboptimal SFA intakes, suggesting that despite the national fortification program, lowincome women of reproductive age in California may be at risk of suboptimal folate status. Furthermore, supplement use and the regular consumption of cereals and bread products were the dominant factors in how well women met their folate needs. We found that $90 \%$ of the women with SFA intakes below the recommended level reported taking folic acid-containing supplements once per month at most. In contrast, $81 \%$ of women with adequate SFA intakes reported taking supplements with folic acid at least twice a month, and most took them more frequently. Of the 13 women who reported taking a folic acid-containing supplement more than once per month but still failed to meet the SFA recommendation, most only took the supplement two to three times per month. They also tended to consume low amounts of foods that are typically fortified with SFA, such as breakfast cereals and bread products. Folate intake from most vegetables appeared to be inadequate to overcome low SFA intake levels.
TABLE 2. Study participants not meeting Institute of Medicine recommendations for folate intake $(n=195)$

\begin{tabular}{lc}
\hline \hline Recommendation & $\begin{array}{c}\text { Women } \\
\text { not meeting } \\
\text { recommendation }\end{array}$ \\
\hline $\begin{array}{l}\text { RDA for adults: } \\
\geq 400 \mu \text { g DFE/day }\end{array}$ & $17(8.7)$ \\
$\begin{array}{l}\text { Special recommendation for } \\
\text { women of childbearing age: }\end{array}$ & $114(58.5)$ \\
$\begin{array}{l}\text { UL for adults: No. with } \mu \mathrm{g} \text { SFA/day } \\
\text { intake }>1,000 \mu \text { SFA/day }\end{array}$ & $3(1.5)$ \\
\hline
\end{tabular}

Similar conclusions can be drawn regarding the 17 women who had total DFE intakes below the RDA. All of the women in this group reported taking supplements once per month at most. In general, women in this group also reported the infrequent consumption of cold breakfast cereal and relatively low intakes of bread products. While some of these women did report the regular consumption of salads and other vegetables, their folate intake from fortified foods was low.

The UL for folate is based on the hypothesis that excessive SFA intake may mask the symptoms of a vitamin B-12 deficiency. Of the 195 study participants, only three had SFA intakes that exceeded the UL. All three of them reported taking a folic acid-containing

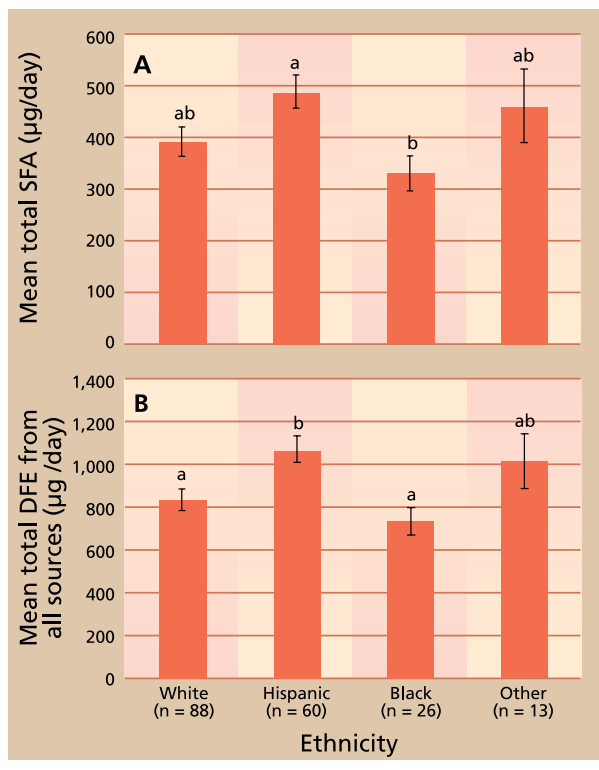

Fig. 1. Mean total (A) synthetic folic acid (SFA) and (B) dietary folate equivalents (DFEs) by ethnicity $(n=187)$. Groups with different letters are significantly different from one another in $A(P<0.05)$ and $B(P<0.01)$. Error bars represent $\pm \mathrm{SE}$. 


\section{Despite the national fortification program, low-income women of reproductive age in California may be at risk of suboptimal folate status.}

multiple vitamin supplement every day, a folic acid or B-complex supplement and/or consuming ready-to-eat breakfast cereal every day, and consuming dark, leafy greens every day. These findings suggest that the risk of folic acid toxicity in this population is limited to a small minority of individuals who consume excessive amounts of SFA daily.

The results demonstrate that folic acid-containing supplements have a considerable impact on total SFA intake in this population. Likewise, in a study of low-income women in the Sacramento area, Clifford et al. (2005) found that the mean $( \pm$ SE) total SFA intake for nonpregnant women of childbearing age was $950 \pm 64 \mu \mathrm{g}$ per day, including a daily supplement of $600 \mu \mathrm{g}$ SFA per day. Before the supplementation period began, the same group of women had a mean SFA intake of $321 \pm 34 \mu \mathrm{g}$ per day, which is below the special recommendation for women of childbearing age.

\section{Ethnicity and folate status}

Our finding that Hispanic women consumed more SFA and total folate than black women is consistent with results from NHANES 1999-2000. However, the finding that Hispanic women also consumed more total folate than white women was surprising. Researchers from the U.S. Centers for Disease Control and Prevention analyzed the NHANES dataset to compare serum and red-blood-cell folate status among women of childbearing age from three ethnic groups (nonHispanic white, non-Hispanic black and Mexican American). They found that non-Hispanic white women had the highest blood folate values, followed by Hispanic women, and then nonHispanic black women (CDC 2002).

There are a few possible explanations for the high folate intakes of Hispanic women in California. One is the possibly higher consumption of certain folate-rich foods, such as beans, fortified tortillas and fortified rice. Although we did not compare the intake of specific food items among ethnic groups, the traditional diet of Hispanic women of Mexican descent is likely to include more of these foods than a more Americanized diet.

Another possible reason for the higher folate intake by Hispanic participants is that they reported higher supplement use than white and black women. For example, $43 \%$ of Hispanic participants reported taking a folic acid-containing supplement at least twice per month, compared to $38 \%$ of white and $36 \%$ of black women. Similarly, 33\% of Hispanic participants reported the daily use of a folic acid-containing supplement, compared to $23 \%$ of white and $15 \%$ of black participants. Although our data do not explain why supplement use is more prevalent among low-income Hispanic women of childbearing age in California, it would be an interesting question to pursue in a future qualitative study.

\section{Targeted nutrition education}

Because more than half of our study participants had total SFA intakes below the Institute of Medicine's recommendation for reducing the risk of neural tube defects, low-income women of childbearing age in California may be at risk of suboptimal folate status. Previous stud-

\section{References}

Bodnar LM, Wisner KL. 2005. Nutrition and depression: Implications for improving mental health among childbearing-aged women. Biol Psychiatry 58:679-85.

Boushey CJ, Beresford SA, Omenn GS, Motulsky AG. 1995. A quantitative assessment of plasma homocysteine as a risk factor for vascular disease. Probable benefits of increasing folic acid intakes. JAMA 274(13):1049-57.

Campbell AK, Jagust WJ, Mungas DM, et al. 2005. Low erythrocyte folate, but not plasma vitamin B-12 or homocysteine, is associated with dementia in elderly Latinos. J Nutr Health Aging 9(1):39-43.

Caudill MA, Le T, Moonie SA, et al. 2001. Folate status in women of childbearing age residing in

Southern California after folic acid fortification. J Am Coll Nutr 20(2):129-34.

[CDC] Centers for Disease Control and Prevention. 2002. Folate status in women of childbearing age, by race/ethnicity — United States, 1999-2000. MMWR 51(36):808-10.

CDC. 2004. Spina bifida and anencephaly before and after folic acid mandate — United States, 19951996 and 1999-2000. MMWR 53(17):362-5. ies have found that socioeconomically disadvantaged groups and some ethnic minorities have limited awareness and understanding of what folate is and why it is important (Kloeblen 1999; Chacko et al. 2003). Targeted nutrition lessons that include folate education could increase intake of this vitamin in low-income women in California.

E.R. Cena is Doctoral Candidate; A. Block Joy is Director, California Food Stamp Nutrition Education (FSNE) program, and Cooperative Extension Specialist; K. Heneman is Post-Doctoral Scholar; and S. Zidenberg-Cherr is Cooperative Extension Specialist; all are in the Department of Nutrition, UC Davis. Carl Keen, UC Davis Department of Nutrition Chair, served as Guest Associate Editor for this manuscript. The USDA Fellowship in Human Nutrition and the FSNE program/USDA provided funding. We thank FSNE program county staff for participant recruitment and data collection: Mary Blackburn, Patty Davidson, Renee Dwyer, Gloria Espinosa-Hall, Margaret Fields, Chutima Ganthavorn, Rogenia Harrison, Sharon Junge, Christine McNamara, Dorothy Smith, Patti Wooten Swanson and Barbara Turner. Our thanks also to Torin Block of Block Dietary Data Systems for scanning the screeners and guidance with questions about the screener. The FSNE program assists food stamp-eligible clients in improving their health and well-being.

Chacko MR, Anding R, Kozinetz CA, et al. 2003. Neural tube defects: Knowledge and preconceptional prevention practices in minority young women. Pediatrics 112:536-42.

Clifford AJ, Noceti EM, Block-Joy A, el at. 2005 Erythrocyte folate and its response to folic acid supplementation is assay dependent in women. J Nutr 135:137-43.

[IOM] Institute of Medicine. 1998. Folate. In: Dietary Reference Intakes for Thiamin, Riboflavin, Niacin, Vitamin B6, Folate, Vitamin B12, Pantothenic Acid, Biotin, and Choline. Washington, DC: Nat Acad Pr. p 196-284.

Jennings E. 1995. Folic acid as a cancerpreventing agent. Med Hypotheses 45(3):297-303.

Kloeblen AS. 1999. Folate knowledge, intake from fortified grain products, and periconceptional supplementation patterns of a sample of low-income pregnant women according to the Health Belief Model. J Am Diet Assoc 99(1):33-8.

[MRC] Medical Research Council Vitamin Study Research Group. 1991. Prevention of neural tube defects: Results of the Medical Research Council Vitamin Study. Lancet 338(8760):131-7. 